\title{
Strong impact of lattice vibrations on electronic and magnetic properties of paramagnetic Fe revealed by disordered local moments molecular dynamics
}

\author{
B. Alling, ${ }^{1,2, *}$ F. Körmann, ${ }^{1,3}$ B. Grabowski, ${ }^{1}$ A. Glensk, ${ }^{1}$ I. A. Abrikosov, ${ }^{2,4}$ and J. Neugebauer ${ }^{1}$ \\ ${ }^{1}$ Max-Planck-Institut für Eisenforschung GmbH, D-402 37 Düsseldorf, Germany \\ ${ }^{2}$ Department of Physics, Chemistry and Biology (IFM), Linköping University, SE-581 83 Linköping, Sweden \\ ${ }^{3}$ Department of Materials Science and Engineering, Delft University of Technology, Mekelweg 2, 2628 CD Delft, Netherlands \\ ${ }^{4}$ Materials Modeling and Development Laboratory, National University of Science and Technology “MISIS”, 119049 Moscow, Russia
}

(Received 23 March 2016; published 9 June 2016)

\begin{abstract}
We study the impact of lattice vibrations on magnetic and electronic properties of paramagnetic bcc and fcc iron at finite temperature, employing the disordered local moments molecular dynamics (DLM-MD) method. Vibrations strongly affect the distribution of local magnetic moments at finite temperature, which in turn correlates with the local atomic volumes. Without the explicit consideration of atomic vibrations, the mean local magnetic moment and mean field derived magnetic entropy of paramagnetic bcc Fe are larger compared to paramagnetic $\mathrm{fcc} F e$, which would indicate that the magnetic contribution stabilizes the bcc phase at high temperatures. In the present study we show that this assumption is not valid when the coupling between vibrations and magnetism is taken into account. At the $\gamma-\delta$ transition temperature $(1662 \mathrm{~K})$, the lattice distortions cause very similar magnetic moments of both bcc and fcc structures and hence magnetic entropy contributions. This finding can be traced back to the electronic densities of states, which also become increasingly similar between bcc and fcc Fe with increasing temperature. Given the sensitive interplay of the different physical excitation mechanisms, our results illustrate the need for an explicit consideration of vibrational disorder and its impact on electronic and magnetic properties to understand paramagnetic Fe. Furthermore, they suggest that at the $\gamma-\delta$ transition temperature electronic and magnetic contributions to the Gibbs free energy are extremely similar in bcc and fcc Fe.
\end{abstract}

DOI: 10.1103/PhysRevB.93.224411

\section{INTRODUCTION}

Iron and its alloys form the material backbone for constructions, vehicles, and tools. The magnetic properties of Fe render it important for electrical motors, generators, and magnetic data storage applications. Besides its industrial relevance, $\mathrm{Fe}$ is also particularly interesting from a fundamental perspective and has attracted enormous experimental and theoretical attention. One of the key problems which has been studied by various research groups for almost half a century is the phase stability and the related bcc-fcc-bcc phase transition sequence observed in Fe with increasing temperature [1-7].

Many studies have been devoted to the description of the magnetic degree of freedom, and in particular the magnetic Gibbs free energy contribution to the phase stability of $\mathrm{Fe}$, in the ferromagnetic and paramagnetic regimes. The employed methods range from phenomenological approaches [1] to quantum mechanical theories [2]. With the advent of high performance computational resources, advanced first-principles techniques can now be applied to study the magnetic and electronic structure of $\mathrm{Fe}$ at elevated temperature. These include the dynamical mean field theory (DMFT) [5,8-14], Hubbard models [15], the dynamical coherent potential approximation [16], extended Heisenberg models based on parameters calculated with density functional theory [17-21], spin cluster expansions [22-24], or spin-fluctuation theory [6]. For an overview of the recent advances in this field see, e.g., Refs. [4,25].

At ambient pressure, the structural phase transitions of $\mathrm{Fe}$ take place in the paramagnetic state, at temperatures $65 \%$

\footnotetext{
*alling@mpie.de
}

$\left(T^{\alpha-\gamma}=1185 \mathrm{~K}\right)$ and $92 \%\left(T^{\gamma-\delta}=1662 \mathrm{~K}\right)$ of the melting temperature $\left(T_{M}=1811 \mathrm{~K}\right)$. Thus, both magnetic disorder and lattice vibrations are relevant for their understanding and for accurate thermodynamic modeling. In recent years the theoretical approaches have been further advanced to study even the coupling between magnetic disorder and lattice vibrations. Among the new approaches are the disordered local moment molecular dynamics (DLM-MD) [26], spin-lattice dynamics [27], DMFT based techniques [28], a spin-wave method [29,30], and the spin-space averaging method [31-35]. All of these methods have revealed the importance of magnetic fluctuations on finite-temperature lattice excitations. The discussion of the role of vibrational entropies to the phase stabilities has further been reignited by recent high temperature phonon measurements [32,36,37].

Most of above works focused on the impact of magnetic and electronic excitations on the atomic motion [28-36]. Much less attention has been paid to the reverse effect, i.e., the impact of atomic motion on the magnetic and electronic properties. Yin et al. [38] studied the effect of lattice vibrations, employing classical molecular dynamics, on the magnetic order-disorder transition in bcc-Fe, and found a small effect on the calculated Curie temperature but a large impact on the entropy change of the transition. On the other hand, for the paramagnetic state of $\mathrm{Fe}$ at the temperatures of relevance for the structural transitions, the effect of lattice vibrations on the magnetic and electronic structure is basically unknown. As subtle energetic differences of only $1 \mathrm{meV} /$ atom are known to be crucial even for a qualitative description of the phase stabilities in $\mathrm{Fe}$ [3,39], the effect of mutual magnetic-vibrational interactions needs to be quantitatively assessed. We address this question by employing the DLM-MD method, where the vibrations are modeled according to ab initio molecular dynamics with 
interatomic forces calculated from density functional theory in a series of rapidly changing disordered magnetic states according to the concept of temporarily broken ergodicity [40]. This allows us to explicitly investigate effects of vibrational excitations in the paramagnetic state and to compare the magnetic and electronic properties of bcc ( $\alpha$ and $\delta)$ and fcc $(\gamma) \mathrm{Fe}$ from $0 \mathrm{~K}$ up to the $\gamma-\delta$ transition temperature.

\section{COMPUTATIONAL DETAILS}

All calculations were performed using density functional theory (DFT) and the projector augmented wave (PAW) method [41], as implemented in the Vienna $A b$ initio Simulation Package (VASP) [42,43]. The generalized gradient approximation (GGA) as formulated by Perdew et al. [44] was used to treat exchange correlation effects. We employed a Fe PAW potential considering the $3 d$ and $4 s$ electronic states as valence. For comparison, selected calculations within the local density approximation (LDA) [45,46] were performed on top of the geometries obtained with GGA. The calculations were based on 125-atom supercells consisting of $5 \times 5 \times 5$ repetitions of the bcc and fcc primitive unit cells. A plane-wave energy cutoff of $350 \mathrm{eV}$ was used. For the molecular dynamics, gamma-point sampling of the $k$ space was employed, but up-sampling to a $2 \times 2 \times 2 k$-point grid was performed to compute accurate local magnetic moments. Convergence tests with a $3 \times 3 \times 3 k$-point grid showed differences in local magnetic moments of less than $0.01 \mu_{B}$ compared to the $2 \times 2 \times 2$ grid.

For each considered temperature, calculations were performed at the corresponding reported experimental [47] lattice parameter or at the values from a quadric interpolation or extrapolation from the experimentally reported values. The total pressure of our DLM-MD simulations reveals for all considered temperatures approximately the same small negative pressure of about $-7 \mathrm{GPa}$, irrespective of the considered crystal structure, fcc or bcc. This is consistent with the well-known underestimation of lattice spacing of bcc Fe within GGA. The temperature-independent constant negative pressure also indicates that theoretical and experimental thermal expansion are very similar.

The paramagnetic state of Fe was modeled by the DLMMD [26] at five temperatures: 300, 1085, 1185, 1425, and $1662 \mathrm{~K}$. This method has been applied previously to paramagnetic materials, e.g., to study the bulk moduli [26], phonon spectra and phase stability [48], alloying effects [49,50], and defects [51] in $\mathrm{CrN}$. To elucidate the separate and combined impacts of magnetic disorder and lattice vibrations, we additionally performed ferromagnetic MD simulations at two selected temperatures, i.e., at 300 and $1085 \mathrm{~K}$, the latter being close to $T_{C}$. We furthermore carried out static calculations without vibrations, without and with magnetic disorder, the latter modeled with the magnetic sampling method, a supercell implementation of the disordered local moments method where in the present case 21 different randomly generated collinear disordered magnetic structures were evaluated and averaged, as described in Ref. [52].

The MD simulations were performed using a time step of $1 \mathrm{fs}$ in a canonical ensemble $(N V T)$. The standard Nosé thermostat [53] as implemented in VASP was used to maintain the temperature and to avoid artificial energy drifts. The corresponding Nosé-mass was set to a 40 time step period. After every $5 \mathrm{MD}$ timesteps, i.e., every $5 \mathrm{fs}$, the magnetic state was rearranged using a random equal probability for spin-up and spin-down moments on each site in the supercell, while the positions and velocities of the atoms were unaltered. Thus the magnetic state was changed often on the phonon timescale. Simulating the magnetic disordered state by collinear disordered spin configurations is assumed to accurately describe the magnetic energetics in the fully magnetically disordered state [40,52] and should thus also provide reliable, effective, magnetically averaged interatomic forces in the adiabatic limit. The MD simulations were carried out for up to $5000 \mathrm{fs}$, of which a 1000 fs equilibration period was allowed in all cases before statistical properties were evaluated. Local magnetic moments were calculated with the PAW-projection procedure of VASP. We note that the exact value of the obtained local magnetic moments in itinerant magnetic systems (such as $\mathrm{Fe}$ ) depends on the chosen sphere radius in which the magnetization density is evaluated. We carefully evaluated different integration radii for the moments between 1.0 and $1.5 \AA$. No relevant changes are found for the investigated temperature dependencies of physical quantities. Further, no influence is observed by varying the initial magnetic moments magnitude (between $1.8 \mu_{B}$ and $2.1 \mu_{B}$ ) or by changing the density and magnetization density mixing coefficients during the electronic minimization. During the 5 ionic steps of MD, between re-initiations with new random magnetic states, less than $1 \%$ of the magnetic moment changed sign. This ensures that the transverse magnetic configuration remains globally disordered with, on average, vanishing short-rangeorder parameters. The theoretical relationship between a DLM based model of the paramagnetic state and explicit many-body theories, e.g., DMFT, is discussed in detailed in Ref. [25]

\section{RESULTS AND DISCUSSION}

\section{A. Average local magnetic moments}

We first consider in Fig. 1(a) the evolution with temperature of the mean value of the magnitude of the local $\mathrm{Fe}$ moments, as they are a key ingredient in many magnetic theories and studies on Fe-based systems, both in historically pioneering studies [54-56] and in recent works (see, e.g., Refs. [3,5,9-14,17,57,58]).

As a reference, we start with ferromagnetic (FM) bcc Fe with the magnetic moments computed at a static lattice. The temperature dependence of the moments is in this case only caused by the thermal lattice expansion and electronic Fermi smearing. In this static FM bcc case, the moments increase linearly from $2.25 \mu_{B}$ at $300 \mathrm{~K}$ to $2.45 \mu_{B}$ at $1662 \mathrm{~K}$, which is a direct consequence of the increased distance between the atoms due to thermal expansion resulting in stronger localization of $d$ electrons. Indeed, the isolated effect of temperature induced electronic smearing is known to be a small, less than $0.1 \mu_{B}$, decrease of the moments in this temperature range [59]. If in addition magnetic disorder is taken into account by means of the DLM method but keeping a static bcc lattice, the temperature-dependent trend originating in thermal expansion remains similar (increase from $2.16 \mu_{B}$ 

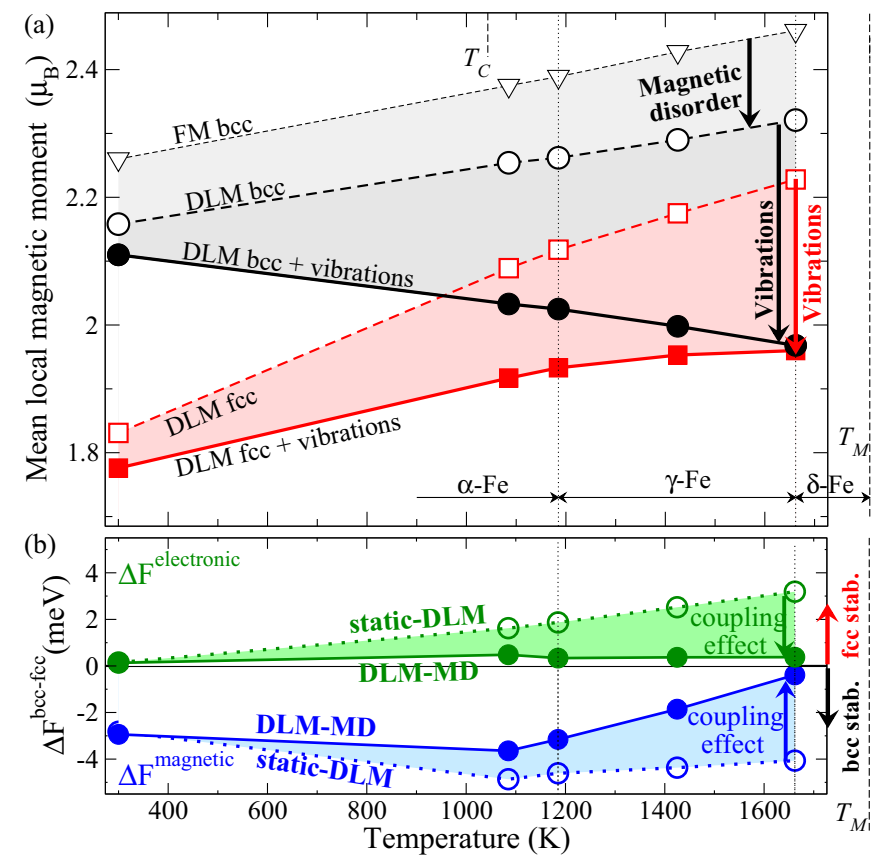

FIG. 1. (a) Mean magnitude of local magnetic moments $\bar{M}$ per atom as a function of temperature in paramagnetic (DLM) bcc and fcc Fe. All data points are calculated at the respective experimental volumes. Electronic contributions are considered for the static and the MD calculations by the corresponding Fermi smearing. The shown MD calculations include effects of vibrationally distorted local geometries on the magnetic moments. For comparison, static FM calculations for bcc are shown. (b) Electronic and magnetic free energy contributions to the bcc-fcc competition in Fe as a function of temperature, with and without explicit vibrational effects.

at $300 \mathrm{~K}$ to $2.33 \mu_{B}$ at $1662 \mathrm{~K}$ ) although the mean magnetic moments are overall lower compared to the ferromagnetic calculations. Due to the volume expansion, the mean moments in fcc Fe in the DLM state calculated on a static lattice are also increasing with temperature $\left(1.85 \mu_{B}\right.$ at $300 \mathrm{~K}$ to $2.24 \mu_{B}$ at $1662 \mathrm{~K}$ ), but are lower compared to the bcc Fe DLM moments. Thus, any analysis of paramagnetic Fe, based on static lattice calculations within a DFT level of theory, will likely conclude that bcc Fe has larger local moments than fcc Fe. Including thermal expansion does not change this, although the difference in moment for the two structures becomes smaller.

Next we investigate the explicit effects of lattice vibrations on the local moment mean values by applying DLM-MD, with atoms vibrating around rather than being fixed on ideal lattice sites. When including lattice vibrations, the mean local moments of DLM bcc Fe decrease with increasing temperature from $2.11 \mu_{B}$ at $300 \mathrm{~K}$ to $1.97 \mu_{B}$ at $1662 \mathrm{~K}$. Thus, the effect of thermal lattice expansion, which increases the bcc moments, is more than compensated when the full vibrational degree of freedom is taken into account. For DLM fcc Fe, explicit vibrations also have a decreasing effect on the mean magnetic moments, but to a smaller degree than for the bcc phase. Thus, taking all effects of vibrations into account, mean magnetic moments still increase with temperature in fcc Fe, from $1.77 \mu_{B}$ at $300 \mathrm{~K}$ to $1.96 \mu_{B}$ at $1662 \mathrm{~K}$. Our analysis shows that at the $\gamma \rightarrow \delta$ transition temperature, the average local magnetic moments of paramagnetic fcc and bcc Fe are almost identical.

In order to verify that the effect of vibrations on magnetic moments observed above is not artificially caused by our use of the GGA approximation for the exchange-correlation energy, we also carried out LDA calculations. The results obtained with LDA show the very same trends with temperature and the same effect of vibrations. The small difference is that, in DLM-MD simulations at $1662 \mathrm{~K}$, the LDA bcc Fe moment, $1.59 \mu_{B}$, is still slightly larger than the LDA fcc Fe moment, $1.52 \mu_{B}$.

It is interesting to compare our findings with the results of Ruban et al. [17] who studied the temperature dependence of bcc Fe moments caused by entropy driven longitudinal spin fluctuations on a static lattice, neglecting explicit lattice vibrations and thermal expansion. It was found that the local bcc Fe moments decreased from $2.01 \mu_{B}$ to $1.85 \mu_{B}$ between 1040 and $1500 \mathrm{~K}$ [17]. This decrease is similar to our findings for bcc Fe once both thermal expansion and explicit vibrational effects are considered, even though the physical mechanisms investigated are different. Ruban et al. [17] sampled the longitudinal magnetic degree of freedom explicitly by constrained spin DFT. In the present work, the longitudinal spin degree is indirectly sampled by vibrationally induced local atomic distortions. The qualitatively similar findings by Ruban et al. and in this work might therefore be explained by the fact that in both cases the energy scale of longitudinal magnetic fluctuations of paramagnetic bcc Fe is probed.

A direct comparison between the absolute values of local moments as obtained in the present work from the magnetization density with the effective moments obtained in DMFT or dynamical coherent potential approximation (CPA) calculations is hindered by the fact that the latter moments are typically obtained indirectly from fitting to a Curie-Weiss-type law of the inverse magnetic susceptibilities. For the temperature trend, Igoshev et al. found a weak decrease of bcc-Fe effective moments with temperature [14]. Kakehashi and Patoary found a weak increase in effective moment with temperature in their dynamical CPA calculations for bcc $\mathrm{Fe}$ [16]. Leonov et al. [5] used GGA+DMFT and calculated the temperature dependence of the square of the instantaneous local moments $\left\langle m_{z}^{2}\right\rangle$. They found that both bcc and fcc $\mathrm{Fe}$ moments increased with temperature [5]. On the other hand, Igoshev et al. performed LDA+DMFT calculations and found that $\left\langle m_{z}^{2}\right\rangle$ of bcc Fe decreased at high temperature [11]. Both the effective local moments and the obtained critical temperature of DMFT calculations depend heavily on the parameters $U$ and $J$ [13]. All DMFT calculations so far have been done assuming an ideal lattice, thus neglecting vibrationally induced disorder.

It is our view that both explicit magnetic and electronic dynamical excitations_-see, e.g., work by Ruban et al. [17] or results on the DMFT level, which are not included here, as well as the vibrational dynamics introduced in the present work-are needed together in a future complete quantitative description of paramagnetic Fe.

In order to asses the impact of vibrations on magnetic free energies of paramagnetic $\mathrm{Fe}$, we use the approximation that the magnetic entropy of a material in the paramagnetic state is given by $S^{\text {mag }}=k_{B} \ln (\tilde{M}+1)$, where $\tilde{M}=\frac{1}{N} \sum_{i}^{N}\left|M_{i}\right|$ denotes the mean local magnetic moments [3]. This expression 
was found to be a good approximation for bcc Fe well above $T_{C}$ [20]. We thus use it to analyze the impact that changes in local magnetic moments have on the relative bcc-fcc phase stability using

$$
\Delta F^{\mathrm{mag}}=-T\left[S_{\mathrm{bcc}}^{\mathrm{mag}}(T)-S_{\mathrm{fcc}}^{\mathrm{mag}}(T)\right] .
$$

The results of static calculations and DLM-MD calculations are shown in blue in Fig. 1(b). There it is seen that the larger local moments in bcc Fe result in a relative stabilization of this phase. In the static calculations, this stabilization is $3 \mathrm{meV} /$ atom at low temperature and increases to $5-4 \mathrm{meV}$ between 1085 and $1662 \mathrm{~K}$. However, when taking into account the coupling between lattice vibrations and magnetic moments, the stabilization diminishes at high temperature as the bcc and fcc local moments become similar. Thus, the explicit vibrational impact effectively removes the magnetic contribution to the stabilization of the bcc phase at the temperature of the $\gamma-\delta$ transition; the actual value of $\Delta F^{\text {mag }}$ that we find in this case is merely $-0.3 \mathrm{meV} /$ atom.

\section{B. Local environment effects on magnetic moments}

Thermally induced vibrations distort the atomic positions and invalidate the model of atoms sitting on identical, ideal lattice sites. In fact, different atoms experience different and dynamically changing local geometries. In particular, for a fixed global volume, each atom possesses a different local volume, which can be evaluated, e.g., via a Voronoi construction [60]. Our DLM-MD and FM-MD simulations give us direct access to the spread in local magnetic moments and their connection to the local geometries as well as to the global magnetic state. The dependence of the individual local magnetic moments on the Voronoi polyhedra volumes is shown in Fig. 2 as a comparison between bcc and fcc Fe at different temperatures. At $300 \mathrm{~K}$, the FM-MD calculated local moments in ferromagnetic bcc Fe shown in Fig. 2(a) are distributed just below the static mean moment (black dashed line). The small horizontal spread reflects the low amplitudes of lattice vibrations at room temperature. When increasing the temperature and considering the paramagnetic bcc phase at 1185 and $1662 \mathrm{~K}$ [panels (c) and (e)] the distribution of calculated local volumes as well as calculated values of magnetic moments are much broader. One can observe that the magnetic moments of atoms with a large local volume qualitatively follow the line describing the static lattice moments. However, the moments of atoms that have a smaller local volume fall below the static lattice line and in addition display a large spread in moment values for the same local volumes. This could be understood if the magnetic moments are sensitive not only to the local volume, which depends on the distance to all the neighbors, but could be heavily suppressed even if only one or two of its neighbors are present at very close distances. The static lattice line corresponds to the maximum shortest neighbor distance for a given volume. Thus, the observed distribution of local moments in the MD simulations, with shorter distance to their closest neighbor for a given local volume, supports such an interpretation.

Considering paramagnetic fcc Fe, right column of Fig. 2, we see similar trends as for bcc Fe. However, it is striking that the DLM-MD simulation of fcc Fe already at $300 \mathrm{~K}$ displays

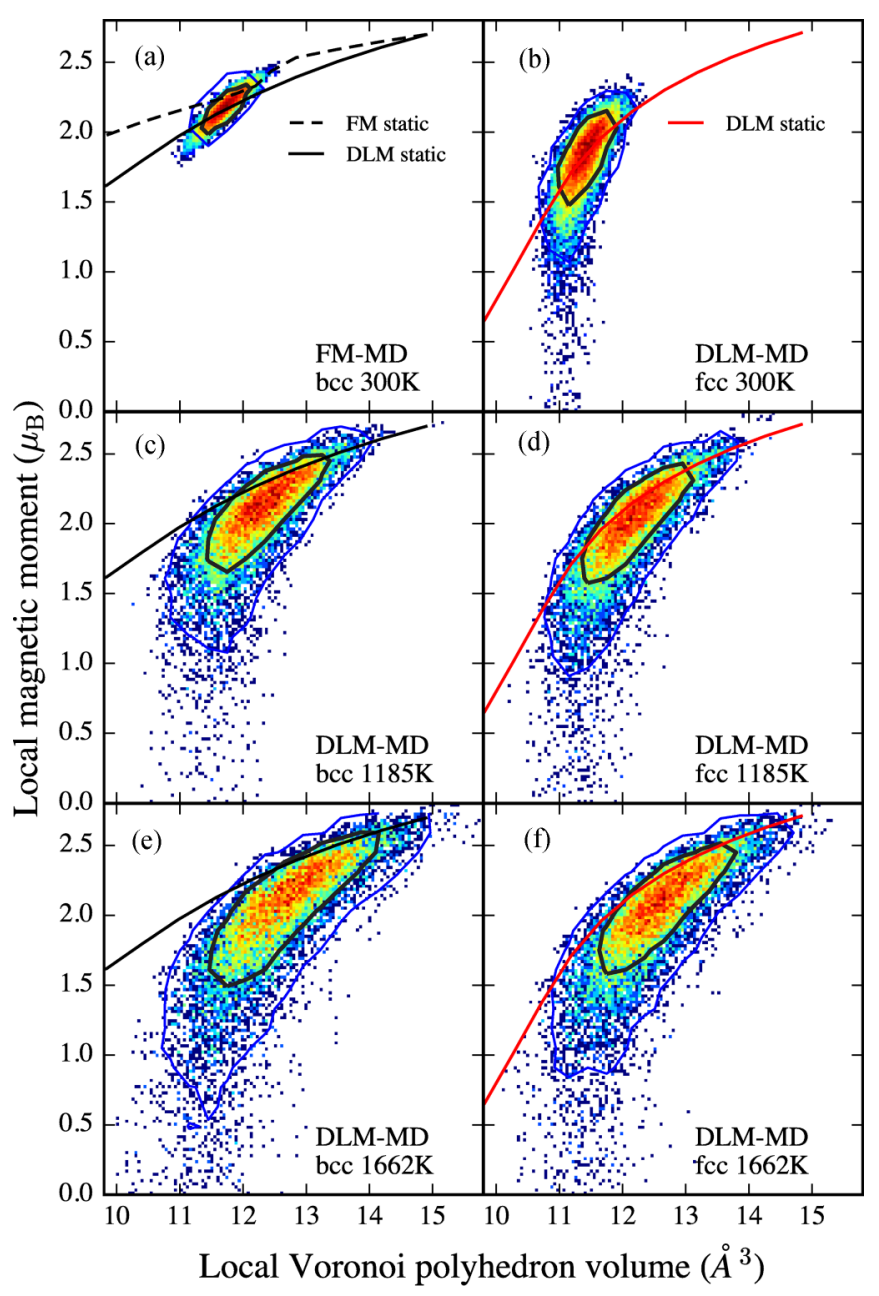

FIG. 2. Contour plots of the distribution of local magnetic moments versus the corresponding local Voronoi polyhedra volumes of the atoms of bcc (left) and fcc (right) Fe at different temperatures obtained with DLM-MD (FM-MD for bcc at $300 \mathrm{~K}$ ) simulations. In all cases, the corresponding temperature-dependent experimental data is used to determine the total volume of the supercell. The mean local moment of static DLM (FM) bcc and fcc Fe as a function of volume is shown as solid (dashed) lines for comparison.

a distinct spread in local moment values, even though the low temperature only induces limited spread in local volumes. This is an illustration that DLM fcc Fe is a quite itinerant magnetic system with sensitive values of the local moments. This is in agreement with the conclusion drawn from DMFT calculations by Igoshev et al. [11].

Two observations can be done using the local moment analysis of Fig. 2. First, the mean moments derived from static DLM calculations are all larger compared to the mean values from the MD runs, i.e., the black/red lines are higher than the mean value in the contour plots. This implies that lattice vibrations weaken the mean local magnetic moments, which is consistent with Fig. 1. A comparison between Figs. 2(c)-2(f) reveals that the corresponding effect is stronger in bcc than in fcc $\mathrm{Fe}$ (see the difference between the scatter plot and the static DLM curve) and results in an effective decrease of the mean local moments of bcc $\mathrm{Fe}$ in the paramagnetic regime. 


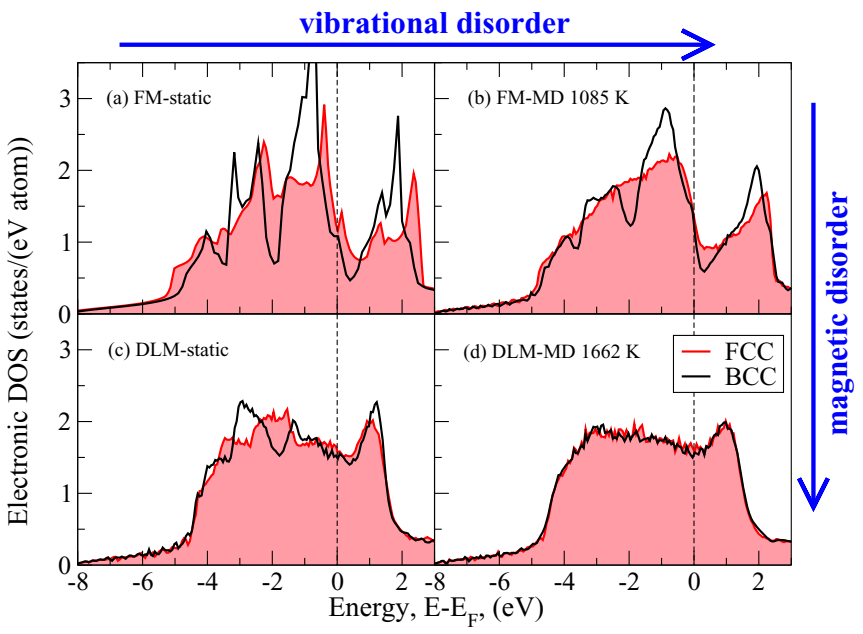

FIG. 3. The calculated total (spin-up + spin-down) electronic DOS of fcc (red shaded) and bcc (black) Fe. (a) Ferromagnetic state in a static lattice, (b) ferromagnetic MD at $1085 \mathrm{~K}$, (c) disordered local moments in static lattice, (d) disordered local moments MD at $1662 \mathrm{~K}$. Calculations are performed at an electronic temperature of $T=0 \mathrm{~K}$ in order to be able to clearly distinguish magnetic from vibrational disorder which could otherwise be hidden by electronic smearing.

Second, if the temperature is increased to $1662 \mathrm{~K}$, the local moment distributions in both crystal structures in Fig. 2 become apparently similar. Thus, the almost identical local mean magnetic moments close to the $\gamma-\delta$ transition observed in Fig. 1(a) are not a mere coincidence of the averaging procedure, but rather a result of the whole distribution of individual moments becoming similar. The global volumes of fcc and bcc Fe at $1662 \mathrm{~K}$ differ by less than $1 \%$ while the first standard deviations of the local volume distributions, caused by vibrations, are much larger: more than $15 \%$ of the total volumes. This could explain a similar behavior of a local property, such as the magnetic moments, even though the global structural differences between bcc and fcc phases remain.

\section{Electronic structure}

We now consider the impact of magnetic disorder and lattice vibrations on the electronic structure of Fe. For this purpose we show in Fig. 3 the total electronic densities of states (DOS) for bcc and fcc Fe computed under different conditions. For the sake of clarity, the electronic temperature is omitted in the derivation of this figure. Figure 3 shows in (a) results of calculations for a system with ferromagnetic order at a static lattice and in (b) the electronic structure for a system with ferromagnetic order including lattice vibrations at $T=1085 \mathrm{~K}$. We show in (c) the electronic structure for DLM magnetic disorder at a static lattice, and in (d) DLM magnetic disorder including lattice vibrations at the $T^{\gamma-\delta}=1662 \mathrm{~K}$. The most significant difference between bcc and fcc electronic DOS is found at $T=0 \mathrm{~K}$ in the ferromagnetic state. Both lattice vibrations (b) and magnetic disorder (c) smear out the DOS to some extent, but distinct characteristics remain, particular close to the Fermi level. However, when both magnetic disorder and high temperature lattice vibrations are considered together in (d), the DOS of the two phases becomes very similar, showing us an electronic origin to the evolution of the magnetic moments discussed above.

Using our static and MD electronic structure calculations we can calculate the electronic free energy contribution to the relative bcc-fcc phase stability. This is done by subtracting the electronic $0 \mathrm{~K}$ energy from the electronic finite temperature free energy for each considered lattice geometry and magnetic state. The difference between bcc and fcc electronic free energies is plotted in green in Fig. 1(b). Under the assumption of static lattice positions, the fcc phase is stabilized by the electronic contributions with $3 \mathrm{meV} /$ atom at $1662 \mathrm{~K}$. On the other hand, when the impact of lattice vibrations is taken into account, this difference disappears, leaving only a $0.3 \mathrm{meV} /$ atom electronic free energy preference for fcc.

To summarize, when the effect of lattice vibrations is considered, the magnitudes of the magnetic and electronic free energy contributions to the structural phase transitions in $\mathrm{Fe}$ are each found to be well below $1 \mathrm{meV} /$ atom at the $\gamma-\delta$ transition and with different sign. If the effects of vibrations are neglected, they are several $\mathrm{meV} /$ atom each, although their combined effect is smaller due to different sign. These findings are in line with the recent suggestions by Neuhaus et al., based on experimental measurements of phonon dispersions, of a very small impact of electronic and magnetic free energies in the stabilization of $\delta$-Fe [37].

\section{CONCLUSION}

We have employed disordered local moments molecular dynamics to study the impact of lattice vibrations on magnetic moments and electronic structure in paramagnetic bcc and fcc Fe. Lattice vibrations have a strong impact on these properties with consequences for phase stabilities. In particular, explicit treatment of the lattice vibrations causes a decrease in the mean magnetic moments in paramagnetic bcc Fe with increasing temperature and significantly reduces the increase of the moments of paramagnetic fcc Fe.

Our work reveals that at the $\gamma-\delta$ phase transition temperature the local magnetic moments as well as the electronic densities of states of paramagnetic bcc and fcc Fe are nearly identical. The relative stabilization of bcc compared to the fcc phase due to the magnetic free energy contributions is found to be strongly suppressed. Also electronic free energy differences between fcc and bcc Fe are suppressed due to vibrations.

If neither electronic nor magnetic contributions play a large role for the high-temperature phase stability of bcc $\delta$-Fe, as shown in Fig. 1(b), one might anticipate that vibrational entropy contributions are the driving force for the $\gamma-\delta$ transition. We note, however, that the Gibbs free energy difference between bcc and fcc Fe in a large temperature window ranging from $T^{\alpha-\gamma}$ to $T^{\gamma-\delta}$ is in the order of $1-2 \mathrm{meV} /$ atom according to empirical data [61] as well as state-of-the-art first-principles calculations [3]. This is likely to rule out the possibility of identifying a single physical mechanism (vibrations, or magnetic or electronic excitations) being responsible for the phase stability sequence, and at the same time reveals the genuine importance of taking into account all excitations, including their mutual interactions. In particular, we have 
shown that the explicit consideration of vibrationally induced lattice disorder is crucial for any first-principles based theory aiming at a quantitative description of paramagnetic iron.

\section{ACKNOWLEDGMENTS}

Tilmann Hickel, Zamaan Raza, and Olle Hellman are acknowledged for useful discussions. B.A. acknowledges financial support by the Swedish Research Council (VR) through Young Researcher Grant No. 621-2011-4417 and International Career Grant No. 330-2014-6336 and by Marie Sklodowska Curie Actions, Cofund, Project INCA 600398, as well as support from the Swedish Government Strategic Research Area in Materials Science on Functional Materials at Linköping University (Faculty Grant SFOMatLiU No 2009 00971). F.K. gratefully acknowledges the Deutsche Forschungsgemeinschaft (DFG) for support via scholarship
KO 5080/1-1. J.N. and B.G. acknowledge financial support by the European Research Council (ERC) under the European Union (EU) Seventh Framework Programme (FP7/20072013)/ERC (Grant No. 290998) and by the ERC under the EU's Horizon 2020 Research and Innovation Programme (Grant No. 639211). I.A.A. is grateful for the support provided by the Swedish Foundation for Strategic Research (SSF) program SRL Grant No. 10-0026, the Swedish Research Council (VR) Grant No. 2015-04391, the Swedish Government Strategic Research Area in Materials Science on Functional Materials at Linköping University (Faculty Grant SFO-Mat-LiU No. 2009 00971), as well as by the Ministry of Education and Science of the Russian Federation (Grant No. 14.Y26.31.0005 and project RFMEFI58715X0023). Calculations were performed utilizing supercomputer resources supplied by the Swedish National Infrastructure for Computing (SNIC) at the PDC and NSC centers.
[1] L. Kaufman, E. Clougherty, and R. Weiss, Acta Metall. 11, 323 (1963)

[2] H. Hasegawa and D. G. Pettifor, Phys. Rev. Lett. 50, 130 (1983).

[3] F. Körmann, A. A. H. Breidi, S. L. Dudarev, N. Dupin, G. Ghosh, T. Hickel, P. Korzhavyi, J. A. Muñoz, and I. Ohnuma, Phys. Status Solidi B 251, 53 (2014).

[4] F. Körmann, T. Hickel, and J. Neugebauer, Curr. Opin. Solid State Mater. Sci. 20, 77 (2016).

[5] I. Leonov, A. I. Poteryaev, V. I. Anisimov, and D. Vollhardt, Phys. Rev. Lett. 106, 106405 (2011).

[6] M. Uhl and J. Kübler, Phys. Rev. Lett. 77, 334 (1996).

[7] C.-S. Lian, J.-T. Wang, and C. Chen, Phys. Rev. B 92, 184110 (2015).

[8] A. I. Lichtenstein, M. I. Katsnelson, and G. Kotliar, Phys. Rev. Lett. 87, 067205 (2001).

[9] I. Leonov, A. I. Poteryaev, V. I. Anisimov, and D. Vollhardt, Phys. Rev. B 85, 020401(R) (2012).

[10] V. I. Anisimov, A. S. Belozerov, A. I. Poteryaev, and I. Leonov, Phys. Rev. B 86, 035152 (2012).

[11] P. A. Igoshev, A. V. Efremov, A. I. Poteryaev, A. A. Katanin, and V. I. Anisimov, Phys. Rev. B 88, 155120 (2013).

[12] A. S. Belozerov, I. Leonov, and V. I. Anisimov, Phys. Rev. B 87, 125138 (2013).

[13] A. S. Belozerov and V. I. Anisimov, J. Phys.: Condens. Matter 26, 375601 (2014).

[14] P. A. Igoshev, A. V. Efremov, and A. A. Katanin, Phys. Rev. B 91, 195123 (2015).

[15] W. Nolting, A. Vega, and T. Fauster, Z. Phys. B. Condens. Matter 96, 357 (1995).

[16] Y. Kakehashi and M. A. R. Patoary, J. Phys. Soc. Jpn. 80, 034706 (2011).

[17] A. V. Ruban, S. Khmelevskyi, P. Mohn, and B. Johansson, Phys. Rev. B 75, 054402 (2007).

[18] F. Körmann, A. Dick, B. Grabowski, B. Hallstedt, T. Hickel, and J. Neugebauer, Phys. Rev. B 78, 033102 (2008).

[19] F. Körmann, A. Dick, T. Hickel, and J. Neugebauer, Phys. Rev. B 79, 184406 (2009)

[20] F. Körmann, A. Dick, T. Hickel, and J. Neugebauer, Phys. Rev. B 81, 134425 (2010).
[21] F. Körmann, A. Dick, T. Hickel, and J. Neugebauer, Phys. Rev. B 83, 165114 (2011).

[22] R. Singer, F. Dietermann, and M. Fähnle, Phys. Rev. Lett. 107, 017204 (2011).

[23] D. Reith, R. Podloucky, M. Marsman, P. O. Bedolla-Velazquez, and P. Mohn, Phys. Rev. B 90, 014432 (2014).

[24] M. Y. Lavrentiev, S. Dudarev, and D. Nguyen-Manh, J. Appl. Phys. 109, 07E123 (2011).

[25] I. A. Abrikosov, A. V. Ponomareva, P. Steneteg, S. A. Barannikova, and B. Alling, Curr. Opin. Solid State Mater. Sci. 20, 85 (2016).

[26] P. Steneteg, B. Alling, and I. A. Abrikosov, Phys. Rev. B 85, 144404 (2012).

[27] P.-W. Ma, C. H. Woo, and S. L. Dudarev, Phys. Rev. B 78 , 024434 (2008).

[28] I. Leonov, A. Poteryaev, Y. N. Gornostyrev, A. Lichtenstein, M. Katsnelson, V. Anisimov, and D. Vollhardt, Sci. Rep. 4 (2014).

[29] A. V. Ruban and V. I. Razumovskiy, Phys. Rev. B 85, 174407 (2012).

[30] A. V. Ruban, V. I. Razumovskiy, and F. Körmann, Phys. Rev. B 89, 179901(E) (2014).

[31] F. Körmann, A. Dick, B. Grabowski, T. Hickel, and J. Neugebauer, Phys. Rev. B 85, 125104 (2012).

[32] F. Körmann, B. Grabowski, B. Dutta, T. Hickel, L. Mauger, B. Fultz, and J. Neugebauer, Phys. Rev. Lett. 113, 165503 (2014).

[33] Y. Ikeda, A. Seko, A. Togo, and I. Tanaka, Phys. Rev. B 90 134106 (2014).

[34] L. Zhou, F. Körmann, D. Holec, M. Bartosik, B. Grabowski, J. Neugebauer, and P. H. Mayrhofer, Phys. Rev. B 90, 184102 (2014).

[35] F. Körmann, P.-W. Ma, S. L. Dudarev, and J. Neugebauer, J. Phys.: Condens. Matter 28, 076002 (2016).

[36] L. Mauger, M. S. Lucas, J. A. Muñoz, S. J. Tracy, M. Kresch, Y. Xiao, P. Chow, and B. Fultz, Phys. Rev. B 90, 064303 (2014).

[37] J. Neuhaus, M. Leitner, K. Nicolaus, W. Petry, B. Hennion, and A. Hiess, Phys. Rev. B 89, 184302 (2014).

[38] J. Yin, M. Eisenbach, D. M. Nicholson, and A. Rusanu, Phys. Rev. B 86, 214423 (2012). 
[39] C. Engin, L. Sandoval, and H. M. Urbassek, Modell. Simul. Mater. Sci. Eng. 16, 035005 (2008).

[40] B. L. Gyorffy, A. J. Pindor, J. Staunton, G. M. Stocks, and H. Winter, J. Phys. F 15, 1337 (1985).

[41] P. E. Blöchl, Phys. Rev. B 50, 17953 (1994).

[42] G. Kresse and J. Furthmüller, Phys. Rev. B 54, 11169 (1996).

[43] G. Kresse and D. Joubert, Phys. Rev. B 59, 1758 (1999).

[44] J. P. Perdew, K. Burke, and M. Ernzerhof, Phys. Rev. Lett. 77, 3865 (1996).

[45] D. M. Ceperley and B. J. Alder, Phys. Rev. Lett. 45, 566 (1980).

[46] J. P. Perdew and A. Zunger, Phys. Rev. B 23, 5048 (1981).

[47] W. B. Pearson and D. Phil, A Handbook of Lattice Spacings and Structures of Metals and Alloys (Pergamon, London, 1958).

[48] N. Shulumba, B. Alling, O. Hellman, E. Mozafari, P. Steneteg, M. Odén, and I. A. Abrikosov, Phys. Rev. B 89, 174108 (2014).

[49] B. Alling, L. Hultberg, L. Hultman, and I. A. Abrikosov, Appl. Phys. Lett. 102, 031910 (2013).

[50] A. Lindmaa, R. Lizárraga, E. Holmström, I. A. Abrikosov, and B. Alling, Phys. Rev. B 88, 054414 (2013).
[51] E. Mozafari, B. Alling, P. Steneteg, and I. A. Abrikosov, Phys. Rev. B 91, 094101 (2015).

[52] B. Alling, T. Marten, and I. A. Abrikosov, Phys. Rev. B 82, 184430 (2010).

[53] S. Nosé, Prog. Theor. Phys. Suppl. 103, 1 (1991).

[54] D. Bagayoko and J. Callaway, Phys. Rev. B 28, 5419 (1983).

[55] V. L. Moruzzi, P. M. Marcus, K. Schwarz, and P. Mohn, Phys. Rev. B 34, 1784 (1986)

[56] F. J. Pinski, J. Staunton, B. L. Gyorffy, D. D. Johnson, and G. M. Stocks, Phys. Rev. Lett. 56, 2096 (1986).

[57] I. A. Abrikosov, A. E. Kissavos, F. Liot, B. Alling, S. I. Simak, O. Peil, and A. V. Ruban, Phys. Rev. B 76, 014434 (2007).

[58] L. Zhang, M. Sob, Z. Wu, Y. Zhang, and G.-H. Lu, J. Phys.: Condens. Matter 26, 086002 (2014).

[59] A. V. Ruban, A. B. Belonoshko, and N. V. Skorodumova, Phys. Rev. B 87, 014405 (2013).

[60] F. Aurenhammer, ACM Comput. Surv. 23, 345 (1991).

[61] Q. Chen and B. Sundman, J. Phase Equilib. 22, 631 (2001). 